\title{
Weed diversity in Maize fields of Mastuj valley, Hindukush range, Pakistan
}

Syed Mukaram Shah ${ }^{1 *}$ and Farrukh Hussain ${ }^{2}$

1. Centre of Plant Biodiversity, University of Peshawar-Pakistan

2. Department of Microbiology and Biotechnology, Sarhad University of Science and Information Technology-

Pakistan

*Corresponding author's email: shahecologistuop@gmail.com

Citation

Syed Mukaram Shah and Farrukh Hussain. Weed diversity in maize fields of Mastuj valley, Hindukush range, Pakistan. Pure and Applied Biology. Vol. 5, Issue 4, pp1044-1050. http://dx.doi.org/10.19045/bspab.2016.50129

\begin{tabular}{llll}
\hline \hline Received: 20/04/2016 & Revised: 19/08/2016 & Accepted: 15/09/2016 & Online First: 26/09/2016
\end{tabular}

\section{Abstract}

Ecological studies in maize (Zea mays L.) fields of Mastuj valley was conducted to record the weeds in three villages. Forty-three 43 species belonging to 19 families were recorded. It included seventeen dicotyledonous, one monocotyledon and one pteridophyte families. Asteraceae was prominent with eight species which was followed by Papilionaceae and Polygonaceae with six and four species. Poaceae and Caryophyllaceae were represented by three species each. Five families had two while nine families had one species. Therophytes were abundant succeeded by geophytes with four and hemicryptophytes with two species. Microphyll (thirteen species) and mesophyll (twelve species) were leading leaf-sizes. Nanophyll had (nine species), macrophyll (eight species) and leptophyll had only one species. Phenologically forty one were in flowering and two species were in dying/post-reproductive stages. The abundance assessment indicated that 18 species were occasional, 9 frequent, 8 rare, 5 common and 3 were abundant. The weeds were classified into fodder (37 spp.), medicinal (13 ssp.), vegetables ( 8 spp.) and oil yielding (1 sp.). Weed management procedures must be practiced to control the weed infusion and invasion. Weeds must be categorized into economic use classes to get additional economic values. Pre-mature hand pulling of whole plant of noxious weeds is recommended. Future researches to conserve the genetic resources through their propagation and distribution is strongly recommended. The traditional cereal crops pattern must be practiced to conserve the vanishing agro plant biodiversity.

Keywords: Mastuj Valley; Maize; Crop; Weeds; Diversity

\section{Introduction}

Mastuj Valley is a high altitude subalpine area in Hindukush Range, District Chitral, Pakistan. Mastuj Valley lies approximately in between $72^{\circ}-0^{\prime}$ and $37^{\circ}-58^{\prime}$ east longitude and $36^{\circ}-2^{\prime}$ and $36^{\circ}-57^{\prime}$ north latitude. It is bounded on the North by Baroghil Pass, on the South by Shandur Pass, on the East by Chumarkhan Pass and on the West by Chitral sub-division. Precipitation in the state of rainfall and snow fall, springs, streams and river channels are source of irrigation. Maize (Zea mays L.) is a monocotyledonous cereal crop which is grown as a spring and summer seasons staple food crop in both plain and hilly rural areas. It needs soil with added OM, adequate irrigation and weeding and thinning. It is cultivated for grain and fodder in the area. White and yellow varieties are met in the 
area (Figure 1, 2). Weed infestation and smut attack are the major threats to maize in the area (Figure 2). Many articles have been published on weeds from maize fields from aboard and Pakistan. Some work on the weed ecology in Mastuj is also available; Hussain et al.[1], Murad et al. [2], Hussain and Murad [3], Hussain et al. [4], Hussain et al. [5], Hussain and Murad [6] and Shah et al.[7]. Vieyra-Odilon \& Vibrans [8], Shah et al. [9], Muhammad et al. [10], Yeganehpoor et al. [11], Janak and Grichar [12] and Dlamini et al. [13] worked on weed ecology. The present study was conducted to further the knowledge about the weeds of this remote area of Hindukush for future workers (Figure 3).

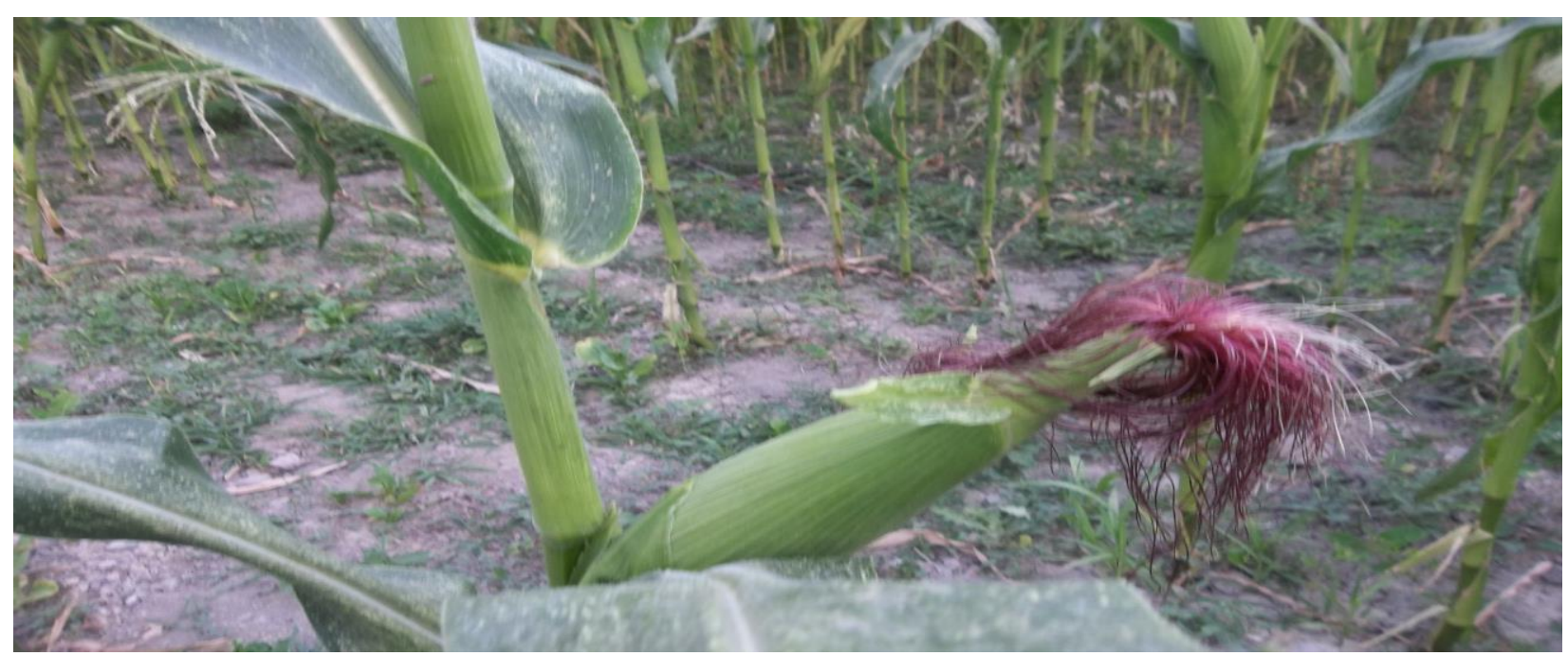

Figure 1. A view of Maize field in Mastuj village showing some weeds

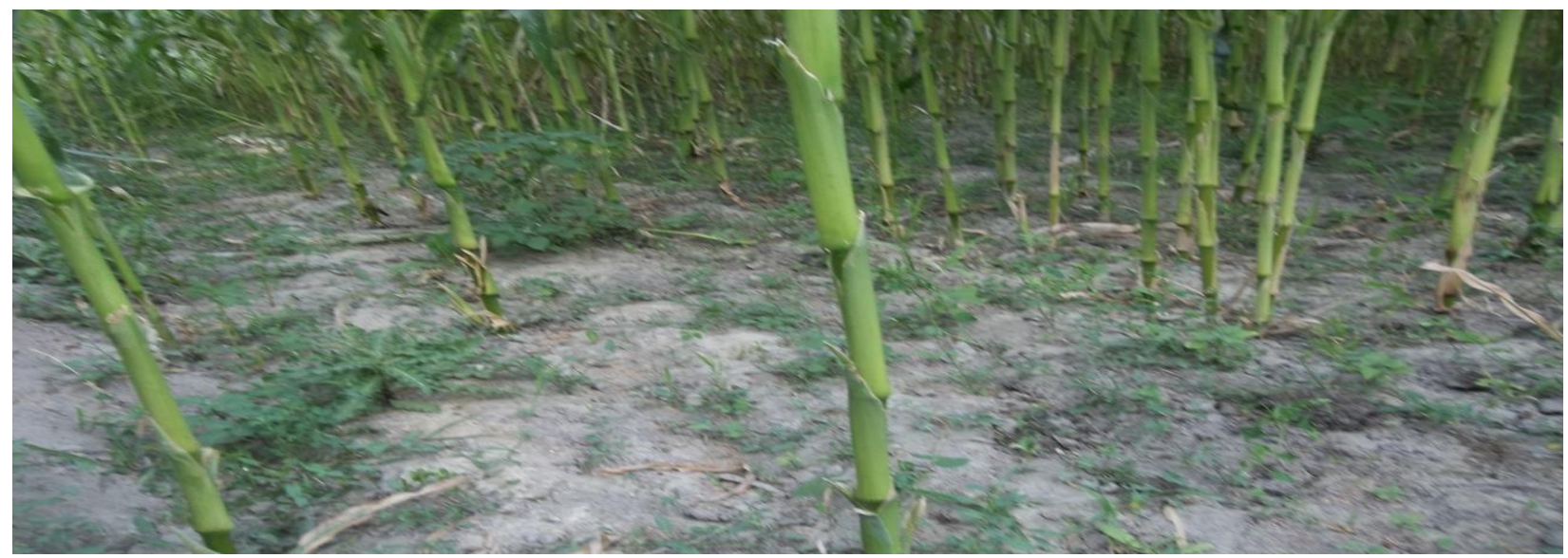

Figure 2. Weeds in Maize field in Mastuj

\section{Materials and methods}

Three villages viz; Mastuj, Tooque and Chinar were surveyed for the presence of weeds during July and August, 2014. The collected weeds were named following Flora of Pakistan [14-16]. Life-forms and leafsizes calculated known after C.C. Raunkiaer
[17] and Hussain [18]. Plants were classified by field observation into flowering and dying phenological stages. Plant species were assigned into various abundance classes based on visual relative species abundance. Ethnobotanical information was 
gathered from the local inhabitants; and also Added voucher specimen number to the collected specimen and deposited in the herbarium.

\section{Results and discussion}

There were 34 species in Mastuj, 32 in Tooque and35 in Chinar thus making a total of 43 species within 19 families. It included 17 dicotyledons, one monocotyledon and one pteridophyte families (Table 1). Asteraceae with eight species (18.60 percent) was dominant, followed by Papilionaceae with six species (13.95 percent) and Polygonaceae possessed four species (9.30 percent). Caryophyllaceae and Poaceae each had three species (6.97 percent). Five families namely: Amaranthaceae, Chenopodiaceae, Lamiaceae, Plantaginaceae and Solanaceae had two species (4.65 percent).Nine families had one species representation. Asteraceae is a leading family in flora of Pakistan and also in Mastuj Valley [1-7]. This supports the present findings. Some of the weeds are common to previous reports in maize fields from the same location $[1,2]$.There were 37 (86.04 percent) therophytes, 4 (9.30 percent) geophytes and two (4.65 percent) hemicryptophytes. Microphylls were the leading leaf-size class (13 spp., 30.23 percent). A similar life form spectrum of weeds $[1,7]$ from Mastuj has been recorded that supports the present findings. It was followed by mesophylls (12 spp., 27.90 percent), nanophylls (9 spp., 20.93 percent), macrophylls (8 spp., 18.60 percent) and supplemented with personal observations. leptophylls (1 sp., 2.32 percent). Previously $[1,7]$ also reported leaf size spectra of weeds of some crops from Mastuj. This agrees with them. Phenological studies indicated that $41(95.34 \%)$ weeds were in reproductive and only two $(4.65 \%)$ were in the post-reproductive stages. Abundance classes revealed that majority of weed species were occasional (18 spp, 41.86\%), followed by frequent $(9 ., 20.93 \%)$, rare (8., $18.60 \%$ ) and common (5., 11.62\%). Only three species $(6.97 \%)$ were abundant (Table 2). Ethnobotanical classification indicated that $37(86.04 \%)$ species were used as fodder, $13(30.23 \%)$ were medicinal, $8(18.60 \%)$ served as vegetables and one $(2.32 \%)$ yielded vegetable oil. Weeds are considered as unwanted plants that grow against the will of man; but some weeds are useful $[8,9]$ as fodder, vegetable, medicinal species as is the case in present study. Cannabis sativa, Eragrostris minor,Helianthus annuus, Nepeta cataria and Papaver somniferum were present in Mastuj village, while Amaranthus cruetiens, Dtura stramonium, Lotus corniculatus and Trifolium resupinatum were found in Chinar village and Cynodon dactylon was observed only in Tooque village (Table 1). Weeds compete, exhibit allelopathy and provide habitats for harmful pathogenic organisms. The yield of maize can be increased by advance agronomic practices including weed control. 
Table 1. Floristic composition and ecological characteristics of weeds of maize fields in Mastuj valley Hindukush range, Pakistan

\begin{tabular}{|c|c|c|c|c|c|c|c|c|c|c|}
\hline \multirow{2}{*}{ S. No. } & \multirow{2}{*}{ Weed species } & \multicolumn{3}{|c|}{ Localities } & \multirow{2}{*}{ Life-form } & \multirow{2}{*}{ Leaf-size } & \multirow{2}{*}{ Phenology } & \multirow{2}{*}{$\begin{array}{c}\text { Abundance } \\
\text { classes }\end{array}$} & \multirow{2}{*}{$\begin{array}{c}\text { Ethnobotanical } \\
\text { uses }\end{array}$} & \multirow{2}{*}{$\begin{array}{c}\text { Voucher } \\
\text { No }\end{array}$} \\
\hline & & Mastuj & Tooque & Chinar & & & & & & \\
\hline & A. $\quad$ Pteridophytes & & & & & & & & & \\
\hline & 1. Equisetaceae & & & & & & & & & \\
\hline \multirow[t]{4}{*}{1.} & Equisetum ramossimum Desf. & - & + & + & $\mathrm{G}$ & $\mathrm{L}$ & Rep & $\mathrm{O}$ & $\mathrm{Fd}$ & SMF01 \\
\hline & B. Angiosperms & & & & & & & & & \\
\hline & Monocotyledons & & & & & & & & & \\
\hline & 2. Poaceae & & & & & & & & & SMF02 \\
\hline 2. & Cynodon dactylon (L.) Pers & - & + & - & $\mathrm{H}$ & Mic & Rep & $\mathrm{F}$ & $\mathrm{Fd}$ & \\
\hline 3. & Eragrostris minor Host. & + & - & - & Th & Mic & Rep & $\mathrm{C}$ & $\mathrm{Fd}$ & SMF03 \\
\hline \multirow[t]{3}{*}{4.} & $\begin{array}{l}\text { Setaria glauca (Retz.) Trinex } \\
\text { Steud. }\end{array}$ & + & + & + & Th & Mic & Rep & $\mathrm{C}$ & $\mathrm{Fd}$ & SMF04 \\
\hline & Dicotyledons & & & & & & & & & \\
\hline & 3. Amaranthaceae & & & & & & & & & \\
\hline 5. & Amaranthus cruetiens L. & - & - & + & Th & Mac & Rep & $\mathrm{R}$ & $\mathrm{Fd}$ & SMF05 \\
\hline \multirow[t]{2}{*}{6.} & Amaranthus reteoflexus L. & + & + & + & Th & Mes & Rep & $\mathrm{R}$ & $\mathrm{Fd}$ & SMF06 \\
\hline & 4. Asteraceae & & & & & & & & & \\
\hline 7. & Artemisia japonica Thunb. & + & + & + & $\mathrm{Th}$ & Mes & Rep & $\mathrm{F}$ & Med & SMFO7 \\
\hline 8. & Cichorium intybus $\mathrm{L}$. & + & + & + & Th & Mes & Rep & $\mathrm{C}$ & $\mathrm{Fd}$ & SMF08 \\
\hline 9. & Cnicus benedictus L. & - & - & + & Th & Mes & Rep & $\mathrm{A}$ & $\mathrm{Fd}$ & SMF09 \\
\hline 10. & Helianthus annuus L. & + & - & - & Th & Mac & Rep & $\mathrm{O}$ & Fd, oil & SMF10 \\
\hline 11. & Matricaria chamomila $\mathrm{L}$. & + & + & + & Th & Mic & Rep & $\mathrm{O}$ & $\mathrm{Fd}$ & SMF11 \\
\hline 12. & Sonchus arvensis Boiss & + & + & + & Th & Mes & Rep & $\mathrm{F}$ & $\mathrm{Fd}$ & SMF12 \\
\hline 13. & Taraxacum officinale Weber. & + & + & + & $\mathrm{Yh}$ & Mac & Rep & $\mathrm{F}$ & $\mathrm{Fd}$ & SMF13 \\
\hline \multirow[t]{2}{*}{14.} & Xanthium strumarium L. & - & + & + & Th & Mac & Rep. & $\mathrm{A}$ & $\mathrm{Fd}$ & SMF14 \\
\hline & 5.Brassicaceae & & & & & & & & & \\
\hline \multirow[t]{2}{*}{15.} & $\begin{array}{c}\text { Capsella bursa-pastoris (L.) } \\
\text { Medic. }\end{array}$ & + & + & + & $\mathrm{Th}$ & Mic & Rep. & $\mathrm{F}$ & $\mathrm{Fd}$ & SMF15 \\
\hline & 6.Cannabaceae & & & & & & & & & \\
\hline \multirow[t]{2}{*}{16.} & Cannabis sativa $\mathrm{L}$. & + & - & - & Th & Mes & Rep & $\mathrm{R}$ & Med & SMF16 \\
\hline & 7.Caryophyllaceae & & & & & & & & & \\
\hline 17. & Arenaria serphyllifolia $\mathrm{L}$. & + & + & + & $\mathrm{Th}$ & Mic & Rep & $\mathrm{O}$ & $\mathrm{Fd}$ & SMF17 \\
\hline 18. & Silene conoidea $\mathrm{L}$. & + & + & + & Th & Mic & Rep & $\mathrm{O}$ & Fd, Veg & SMF18 \\
\hline \multirow[t]{2}{*}{19.} & Vaccaria pyramidata Medik. & + & + & - & Th & $\mathrm{N}$ & Rep. & $\mathrm{O}$ & $\mathrm{Fd}$ & SMF19 \\
\hline & 8.Chenopodiaceae & & & & & & & & & \\
\hline 20. & Chenopodium album $\mathrm{L}$. & + & + & + & Th & Mic & Rep & $\mathrm{C}$ & $\mathrm{Fd}$ & SMF20 \\
\hline \multirow[t]{2}{*}{21.} & Chenopodium botrys L. & + & + & + & Th & $\mathrm{N}$ & Rep & $\mathrm{C}$ & $\mathrm{Fd}$ & SMF21 \\
\hline & 9.Convolvulaceae & & & & & & & & & \\
\hline 22. & Convolvulus arvensis L. & + & + & + & $\mathrm{Th}$ & Mic & Rep & $\mathrm{O}$ & $\mathrm{Fd}$ & SMF22 \\
\hline
\end{tabular}




\begin{tabular}{|c|c|c|c|c|c|c|c|c|c|c|}
\hline & 10.Euphorbiaceae & & & & & & & & & \\
\hline 23. & Euphorbia peplus L. & - & + & + & Th & $\mathrm{N}$ & Rep & $\mathrm{R}$ & $\mathrm{Fd}$ & SMF23 \\
\hline & 11.Labiatae & & & & & & & & & \\
\hline 24. & Mentha longifolia (L.) Huds & + & + & + & $\mathrm{G}$ & Mic & Rep & $\mathrm{O}$ & Fd, Med & SMF24 \\
\hline 25. & Nepeta cataria $\mathrm{L}$. & + & - & - & Th & Mes & Rep & $\mathrm{R}$ & Fd, Med & SMF25 \\
\hline & 12.Malvaceae & & & & & & & & & \\
\hline 26. & Malva neglecta Wall. & + & + & + & Th & Mic & Rep. & $\mathrm{O}$ & Fd, Med, Veg & SMF26 \\
\hline & 13.Papaveraceae & & & & & & & & & \\
\hline 27. & Papaver somniferum L. & + & - & - & Th & Mac & Post-Rep & $\mathrm{R}$ & Med & SMF27 \\
\hline & 14.Papilionaceae & & & & & & & & & \\
\hline 28. & Glycyrrhiza glabra L. & + & + & + & $\mathrm{G}$ & Mes & Rep & $\mathrm{A}$ & Fd, Med & SMF28 \\
\hline 29. & Lotus corniculatus L. & - & - & + & Th & $\mathrm{N}$ & Rep & $\mathrm{F}$ & $\mathrm{Fd}$ & SMF29 \\
\hline 30. & Medicago sativa $\mathrm{L}$. & + & + & + & $\mathrm{H}$ & $\mathrm{N}$ & Rep & $\mathrm{F}$ & Fd, Veg & SMF30 \\
\hline 31. & Trifolium repens $\mathrm{L}$. & + & + & + & Th & $\mathrm{N}$ & Rep & $\mathrm{F}$ & $\mathrm{Fd}$ & SMF31 \\
\hline 32. & Trifolium resupinatum $\mathrm{L}$. & - & - & + & Th & Mic & Rep & $\mathrm{O}$ & Fd, Veg & SMF32 \\
\hline 33. & Vicia sativa $\mathrm{L}$. & + & + & - & Th & Mic & Rep & $\mathrm{O}$ & $\mathrm{Fd}$ & SMF33 \\
\hline & 15.Plantaginaceae & & & & & & & & & \\
\hline 34. & Plantago lanceolata $\mathrm{L}$. & + & + & + & Th & Mes & Rep & $\mathrm{F}$ & Fd, Med & SMF34 \\
\hline 35. & Plantago major Aitch. & + & + & + & $\mathrm{G}$ & Mac & Rep. & $\mathrm{O}$ & Fd, Med & SMF35 \\
\hline & 16.Polygonaceae & & & & & & & & & \\
\hline 36. & Polygonum aviculare $\mathrm{L}$. & + & + & + & Th & $\mathrm{N}$ & Rep & $\mathrm{O}$ & $\mathrm{Fd}$ & SMF36 \\
\hline 37. & $\begin{array}{l}\text { Fallopia dumetorum (Linn.) } \\
\text { Holub }\end{array}$ & + & + & + & $\mathrm{Th}$ & Mes & Rep & $\mathrm{R}$ & Fd, Veg & SMF37 \\
\hline 38. & Persicaria maculosa Gray. & + & + & + & Th & Mes & Post-Rep & $\mathrm{O}$ & $\mathrm{Fd}$ & SMF38 \\
\hline 39. & Rumex longifolius DC. & + & - & + & Th & Mac & Post-Rep & $\mathrm{O}$ & Fd, Med, Veg & SMF39 \\
\hline & 17.Portulacaceae & & & & & & & & & \\
\hline 40. & Portulaca oleracea L. & + & + & + & Th & $\mathrm{N}$ & Rep & $\mathrm{R}$ & Med, Veg & SMF40 \\
\hline & 18.Rubiaceae & & & & & & & & & \\
\hline 41. & Gallium aparine L. & + & + & + & $\mathrm{Th}$ & $\mathrm{N}$ & Rep & $\mathrm{O}$ & $\mathrm{Fd}$ & SMF41 \\
\hline & 19. Solanaceae & & & & & & & & & \\
\hline 42. & Datura stramoniumL. & - & - & + & Th & Mac & Rep & $\mathrm{O}$ & Med & SMF42 \\
\hline 43. & Solanum nigrum L. & + & + & + & Th & Mes & Rep. & $\mathrm{O}$ & Med, Veg & SMF43 \\
\hline
\end{tabular}

Key: 1. Life-form classes: Th. Therophyte., G. Geophyte., H. Hemicryptophyte. 2. Leaf-size classes: L. Leptophyll., N. Nanophyll., Mic. Microphyll., Mes. Mesophyll., Mac. Macrophyll. 3. Phenological stages: Rep. Reproductive., Post-Rep. Post Reproductive. 4. Abundance classes: A. Abundant., C. Common., F. Frequent., O. Occasional. R. Rare. 5. Economic values: Fd. Fodder., Med. Medicinal., Veg. Vegetables Oil 
Table 2. Summary of biological spectra and economic uses of weeds of maize in Mastuj valley, Pakistan.

\begin{tabular}{|c|c|c|c|}
\hline S.No. & Ecological Parameters & No. of Species & Percentage (\%) \\
\hline A. & Life-form classes & & \\
\hline i. & Therophytes: Th & 37 & 86 \\
\hline ii. & Geophytes: G & 04 & 09 \\
\hline iii. & Hemicryptophytes: $\mathrm{H}$ & 02 & 04 \\
\hline \multicolumn{2}{|l|}{ Total } & 43 & 99 \\
\hline B. & Leaf-size classes & & \\
\hline i. & Microphyll: Mic & 13 & 30 \\
\hline ii. & Mesophyll: Mes & 12 & 27 \\
\hline iii. & Nanophyll: N & 09 & 20 \\
\hline iv. & Macrophyll: Mac & 08 & 18 \\
\hline & Leptophyll: L & 01 & 02 \\
\hline \multicolumn{2}{|l|}{ Total } & 43 & 97 \\
\hline C. & Phenological Stage & & \\
\hline i. & Reproductive & 41 & 95 \\
\hline ii. & Post-Rep. & 02 & 04 \\
\hline \multicolumn{2}{|l|}{ Total } & 43 & 99 \\
\hline D. & Abundance Classes & & \\
\hline i. & Occasional & 18 & 41.86 \\
\hline ii. & Frequent & 09 & 20.93 \\
\hline iii. & Rare & 08 & 18.60 \\
\hline iv. & Common & 05 & 11.62 \\
\hline v. & Abundant & 03 & 6.97 \\
\hline \multicolumn{2}{|l|}{ Total } & 43 & 99.98 \\
\hline D. & Economic use Classification & & \\
\hline i. & $\begin{array}{l}\text { Fodder } \\
\text { Medicinal }\end{array}$ & $\begin{array}{l}37 \\
13\end{array}$ & $\begin{array}{l}86.04 \\
30.23\end{array}$ \\
\hline iii. & Vegetables & 08 & 18.60 \\
\hline iv. & Edible oil yielding & 01 & 2.32 \\
\hline
\end{tabular}

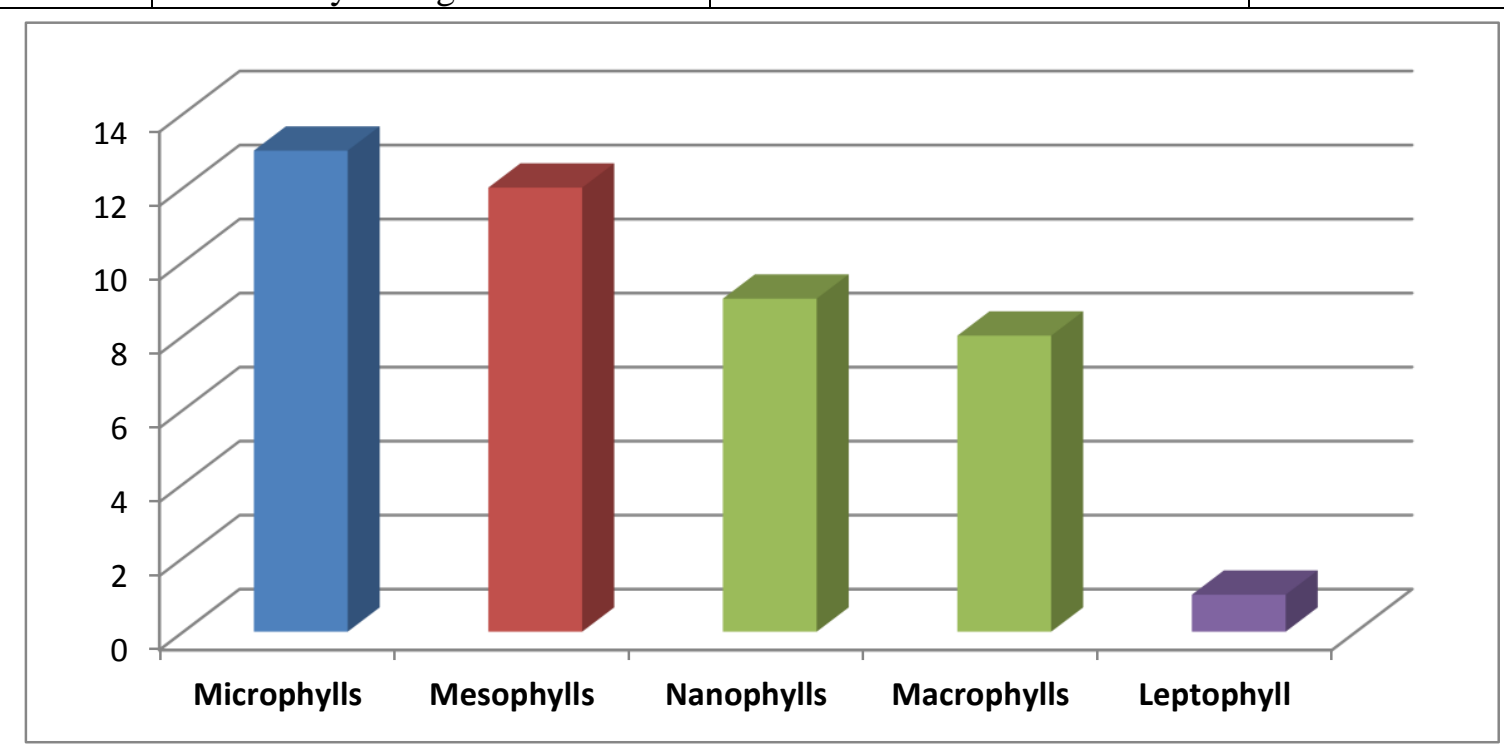

Figure 3. Leaf-size spectrum of weeds in maize fields of Mastuj valley 


\section{Conclusion and recommendations}

It is concluded that the research area is suitable for maize cultivation due to its fertility, availability of water and farmer hard working. Hand pulling of weeds at prereproductive stage is recommended.

\section{Author's contributions}

Conceived and designed the experiments: SM Shah, Performed the experiments: SM Shah, Analyzed the data: F Hussain, Provided reagents: $F$ Hussain, Wrote the paper: SM Shah.

\section{References}

1. Hussain F, Murad A \& Marwat Q (1994). Distribution and population of weeds in the maize fields of Mastuj, District Chitral. Pak J Weed Sci 7: 42-48.

2. Murad A, Hussain F, Marwat Q \& Muhammad Z (1995). Floristic composition, Life-form and Leaf-size spectra of some weeds of wheat, maize and potato fields of Mastuj, District Chitral. Pak J Pl Sci 1: 153-165.

3. Hussain F \& Murad A (2004). Weed communities in the potato fields of Mastuj, District Chitral. Sci Khyber 17:201-206

4. Hussain F, Durrani MJ, Murad A \& Sanaullah P (2004). Distribution of some weeds in the potato fields of Mastuj, District Chitral, and Pakistan. Pak J Pl Sci 10(1): 25-29.

5. Hussain F, Murad A \& Durrani MJ (2004). Weed communities in the wheat fields of Mastuj, District Chitral, Pakistan. Pak J Weed Sci Res 10:101108.

6. Hussain F \& Murad A (2004). Weed communities in the potato fields of Mastuj, District Chitral. Scientific Khyber 17:201-206

7. Shah SM, Ullah A \& Hadi F (2014). Ecological characteristics of weed flora in the wheat crop of Mastuj valley,
District Chitral, KP, Pakistan. Pak J Weed Sci Res 20(4): 479-487.

8. Vieyra-Odilon L \& Vibran H (2001). Weeds as crops: The value of maize field weeds in the valley of Toluca, Mexico. Economic Bot 55(3): 426-443.

9. Shah SRU, Qasim M, Khan IA \& Shah SAU (2006). Study of medicinal plants among weeds of wheat and maize in Peshawar region. Pak J Weed Sci Res 12(3): 191-197.

10. Muhammad S, Khan ZUD \& Cheema TA (2009). Distribution of weeds in wheat, maize and potato fields of Tehsil Gojra, District Toba Tek Singh, Pakistan. Pak J Weed Sci Res 15(1): 91-105.

11. Yeganehpoor F, Salmasi SZ, Abedi G, Samadiyan F \& Beyginiya V (2016). Effect of cover crops and weed management on corn yield. J Saudi Soc Agri Sci 14(2): 178-181.

12. Janak TW \& Grichar WJ (2016). Weed control in corn (Zea mays L.) as influenced by preemergence herbicides. Int J Agro 9.

13. Dlamini TM, Banda HRM \& Edje OT (2016). Evaluation of the efficacy of selected herbicides on weed biomass control and maize (Zea mays L.) yield production in two agroecological zones in Swaziland. Amer J Agri \& Forestry 4(4): 75-85.

14. Nasir E \& Ali SI (1970-1991). Flora of Pakistan.

15. Ali SI \& Nasir E (1991-1993). Flora of Pakistan.

16. Ali SI \& Qaiser M (1993-2015). Flora of Pakistan.

17. Raunkiaer CC (1934). The life-form of plant and statistical plant geography. Clarendon Press, Oxford.

18. Hussain F (1989). Field and Laboratory Manual of Plant Ecology. UGC, Islamabad. 